\title{
Upland rice and lowland rice exhibited different PIP expression under water deficit and ABA treatment
}

\author{
Hong-Li Lian ${ }^{1}$, Xin Yu ${ }^{1}$, David Lane ${ }^{2}$, Wei-Ning Sun ${ }^{1}$, Zhang-Cheng Tang ${ }^{1}$, Wei-Ai Su${ }^{1}$ \\ ${ }^{1}$ Institute of Plant Physiology and Ecology, Shanghai Institutes for Biological Sciences, Chinese Academy of Sciences, 300 Fenglin \\ Road, Shanghai 200032, China, ${ }^{2}$ Agriculture and Agri-Food Canada, Pacific Agri-Food Research Centre, Summerland, B.C., VOH- \\ 1Z0, Canada
}

Aquaporins play a significant role in plant water relations. To further understand the aquaporin function in plants under water stress, the expression of a subgroup of aquaporins, plasma membrane intrinsic proteins (PIPs), was studied at both the protein and mRNA level in upland rice (Oryza sativa L. cv. Zhonghan 3) and lowland rice (Oryza sativa L. cv. Xiushui 63 ) when they were water stressed by treatment with $20 \%$ polyethylene glycol (PEG). Plants responded differently to $20 \%$ PEG treatment. Leaf water content of upland rice leaves was reduced rapidly. PIP protein level increased markedly in roots of both types, but only in leaves of upland rice after $10 \mathrm{~h}$ of PEG treatment. At the mRNA level, OsPIP1;2, Os$P I P 1 ; 3, O S P I P 2 ; 1$ and $O S P I P 2 ; 5$ in roots as well as $O S P I P 1 ; 2$ and $O S P I P 1 ; 3$ in leaves were significantly up-regulated in upland rice, whereas the corresponding genes remained unchanged or down-regulated in lowland rice. Meanwhile, we observed a significant increase in the endogenous abscisic acid (ABA) level in upland rice but not in lowland rice under water deficit. Treatment with $60 \mu \mathrm{M}$ ABA enhanced the expression of OsPIP 1;2, OsPIP2;5 and OsPIP2;6 in roots and OsPIP1;2, OsPIP 2;4 and $O s P I P 2 ; 6$ in leaves of upland rice. The responsiveness of $P I P$ genes to water stress and ABA were different, implying that the regulation of $P I P$ genes involves both ABA-dependent and ABA-independent signaling pathways during water deficit.

Cell Research (2006) 16:651-660. doi: 10.1038/sj.cr.7310068; published online 13 Jun 2006

Keywords: aquaporin, gene expression, PIP, rice, water deficit

\section{Introduction}

Aquaporins, which belong to the highly conserved major intrinsic protein (MIP) family, play an essential role in plant water transport [1-3]. Various molecular and cellular mechanisms underlying aquaporin regulation in a challenging environment has been well documented. These mechanisms include three basic pathways: changes in aquaporin gene transcription rate and protein abundance, the subcellular localization of aquaporin and the gating of

Correspondence: Wei-Ai Su

Tel: +86-21-54924245; Fax: +86-21-54924015;

E-mail: zstressc@online.sh.cn

Received 12 Nov 2005; revised 23 Jan 2006; accepted 17 Feb 2006; published online 13 Jun 2006 water channels [4]. The expression of aquaporins, at mRNA level, has been the focus of many published studies.

One subgroup of MIPs, the plasma membrane intrinsic proteins (PIPs), which are localized in cellular plasma membrane, has been extensively investigated at the transcriptional level under water deficit/drought treatment. Different PIP genes exhibit different expression patterns. Water deficit was shown to enhance the expression of rice OsPIP $1 a$ and OsPIP2a [5], tomato TRAMP [6], Nicotiana excelsior NeMip2 and NeMip3 [7] and radish RsPIP2 [8], but reduced Nicotiana glauca NgMIP4 [9] and rice $R W C 1$ [10]. In a recent study, both up- and down-regulation of PIP expression were observed under water deficit induced by mannitol in Arabidopsis [11]. The expression of AtPIP1;5, AtPIP 2;2, AtPIP 2;3 and AtPIP2;6 was down-regulated, whereas AtPIP 1;3, AtPIP1;4,AtPIP 2; 1 and AtPIP2; 5 was up-regulated in both the roots and aerial parts of the plants. 
The expression level of AtPIP1;1 increased in the aerial parts during the $12 \mathrm{~h}$-drought treatment, but decreased in roots. In another study, similar expression regulation in AtPIPs was observed in response to drought [12]. However, due to different experimental conditions, some $P I P$ s were different regulated. For example, AtPIP1;3 and AtPIP2;1 were down-regulated by drought. The above results illustrate that the PIP expression pattern under water deficit is a complicated response and the mechanisms underlying the regulation of PIP expression under water deficit are still elusive.

Abscisic acid (ABA) accumulation in stressed plants was found to be protective against drought damage, causing stomata closure that reduces water loss via transpiration $[13,14]$, and increasing hydraulic conductance, promoting water movement from roots to leaves $[15,16]$. In addition, ABA plays an important role in mediating the gene expression in response to water deficit $[17,18]$. It was suggested that water deficit triggered the up-regulation of PIP genes in ABA-dependent and ABA-independent manner $[11,19,20]$. Verification of a similar mechanism in rice would contribute to a greater understanding of the regulation of PIPs.

In our previous study, upland rice and lowland rice showed different mechanisms of drought resistance and diverse expression patterns of one PIP gene, $R W C 3$ (Os$P I P 1 ; 3)$ under water deficit [21]. In the present study, upland rice and lowland rice were initially observed to have different $P I P$ expression patterns at both mRNA and protein levels and had different ABA contents as a result of $20 \%$ polyethylene glycol (PEG). Based on the comparative analysis of the expression of the whole PIP subgroup at the mRNA level between upland rice and lowland rice treated with PEG or exogenous ABA, the potential role of ABA in the regulation of PIP expression is proposed.

\section{Materials and Methods}

\section{Plant material and growth condition}

Physiological experiments were performed using lowland rice (Oryza sativa L. spp. japonica cv. Xiushui 63) and upland rice (Oryza sativa $\mathrm{L}$. spp. indica $\mathrm{cv}$. Zhonghan 3). After germinating in the dark for $3 \mathrm{~d}$ at $28^{\circ} \mathrm{C}$, all seedlings were grown in nutrient solution [22] at a photon flux density of $300-350 \mathrm{~mol} \mathrm{~m}^{-2} \mathrm{~s}^{-1}, 60-80 \%$ relative humidity, $12 \mathrm{~h} / 12 \mathrm{~h}$ day-night cycle at $28^{\circ} \mathrm{C}$ in a phytotron. Four-week-old plants were used for the water deficit treatments, the nutrient solution contained 20\% PEG 6000 giving $-0.82 \mathrm{MPa}$ of stress determined by a Vapor Pressure Osmometer 5520 (Wescor, USA). For the ABA treatment, $60 \mu \mathrm{mol} \mathrm{L}{ }^{-1} \mathrm{ABA}$ solution was prepared by dissolving the crystals in $100 \mu \mathrm{L}$ of ethanol and then adding it to the nutrient solution. The final concentration of ethanol in the nutrient was 100 $\mu \mathrm{L} / \mathrm{L}$. The entire experiments were repeated three times.

\section{Leaf water status and osmotic potential}

Leaf water status was assayed by measuring the fresh weight (FW) and dry weight (DW) of the leaves. DW was obtained after drying the leaves at $100{ }^{\circ} \mathrm{C}$ for $5 \mathrm{~h}$. Leaf water content (WC) was calculated using the following formula:

$\mathrm{WC}(\%)=(\mathrm{FW}-\mathrm{DW}) \times 100 / \mathrm{FW}$

The osmotic potential was measured with a Vapor Pressure Osmometer 5520 (Wescor, USA). Leaves of PEG-treated and control seedlings were harvested and put in a syringe and frozen in a freezer overnight. The leaf juice was squeezed and then the osmotic potential was measured.

Preparation of plasma membranes and immunoblot analysis

Plasma membrane fractions were prepared by aqueous two-phase partitioning as described by Ohshima et al. [23]. Protein concentration was measured according to Bradford [24]. Western blots were carried out using anti-PIP antibody [23] following the standard method according to Sambrook et al. [25]. The PIP antibody was raised against N-terminal common sequence "KDYNEPPPAPLFEPGELSSWS", among six radish PIPs including three PIP1s and three PIP2s. The antibody has broader specificity and was able to detect the PIPs in graptopetalum and radish [23]. We compared all the rice PIP proteins and found high conservation in this region. Standard procedures for SDS-PAGE in $12 \%$ polyacrylamide gels were used and proteins were transferred to nitrocellulose. The anti-PIP antibodies [23] were 1:500 diluted for immunoblotting. The primary antibodies were detected with goat anti-rabbit IgG-HRP (1:1000), and the signal was detected by chemiluminescence method (West Pico West Blotting, Pierce, IL, USA).

\section{Extraction of RNA and first-strand cDNA synthesis}

Total RNA was extracted from roots and leaves using RNArose reagent (Watson, China). The residual genomic DNA was removed by DNA-free ${ }^{\mathrm{TM}}$ Kit (Ambion, USA). The concentration of RNA was accurately quantified by spectrophotometric measurements. The cDNA was synthesized using oligo-(dT) $)_{18}$ primer and ReverTra Ace M-MLV RTase (Toyobo, Osaka, Japan) according to the manufacture's recommendation. The cDNA mixtures were diluted to $60 \mu \mathrm{L}$ with sterile $\mathrm{ddH}_{2} \mathrm{O}$ and $1.8 \mu \mathrm{L}$ were used as template for real-time PCR in a total volume of $20 \mu \mathrm{L}$.

\section{Primers and TaqMan-MGB probes}

The primers and TaqMan-MGB probes were designed using Primer Express 2.0 software (Applied Biosystems-Perkin-Elmer, USA) according to the sequences of rice PIP genes from Sakurai et al. [26]. Ubiquitin (NCBI accession number: D12629) was used as internal control. The sequence of PCR primers and TaqMan-MGB probes are shown in Table 1. All the primers and probes were synthesized by Shanghai GeneCore Company (Shanghai, China). The probes were labeled with the fluorescent reporter dye 6-carboxyfluoroscein on the $5^{\prime}$-end, and with the fluorescent quencher dye 6-carboxytetramethylrhodamine (TAMRA) and MGB on the 3 '-end. They formed extremely stable duplexes with single-stranded DNA targets, allowing shorter probes to be designed. All PCR primers were designed to produce the PCR products of about $70 \mathrm{bp}$ in length.

\section{Real-time PCR}

TaqMan-MGB real-time PCR was performed in PRISM 7700 (ABI, USA) using Real-time PCR Master Mix (Toyobo, Japan). 
Table 1 Sequence of the primers and TaqMan-MGB probes for real-time RT-PCR

\begin{tabular}{llll}
\hline Name & Probe & Forward primer & Reverse primer \\
\hline OsPIP $1 ; 1$ & TGTAGTTGCCAGTGGCT & TACATGGGCAATGGCGGT & CAAGACCGTCACCCTTGGTG \\
OsPIP $1 ; 2$ & ACCGTCCTCACCGTCAT & GGCCACCTTCCTCTTCCTTTAC & GCGCACTTGGAGGTGGAGT \\
OsPIP $; ; 3$ & CTCCTCCACCTCGAACA & AAGGACTACCGGGAGCCG & GGTAGAACGACCACGACGTCA \\
OsPIP $2 ; 1$ & TCGGCCTCGTCAAGG & AGTGCCTCGGCGCCAT & CGTACCTGTTGAAGTAGGCGCT \\
OsPIP $2 ; 2$ & CTGATCAGGGCGGTGC & TTCGGGCTGTTCCTGGC & GGCACTGCGCGATGATGTA \\
OsPIP $2 ; 3$ & AAGAGGAGCAAGCACGG & TCTTCTCCGCCACCGACC & AACACCGCGAACCCAATTG \\
OsPIP $; ; 4$ & ACTCACATGTTCCCGTGTT & ACCGATCCCAAGCGCAA & GCGAACCCGATTGGCAG \\
OsPIP $2 ; 5$ & CGACCATCCCCGTCAC & CCGTGTTCATGGTGCACCT & CCGGGTTGATGCCGGT \\
OsPIP $2 ; 6$ & ACACCGTCTTCTCCGC & GAAATCATCGGCACCTTCGT & GGGCATTGCGCTTTGG \\
OsPIP $2 ; 7$ & CTGTACCGTGCCCTC & ACGAGCGAGCTGGGTAAGTG & ATGAGCGTCGCCATGAACTC \\
OsPIP $2 ; 8$ & CTGCTGTTGGTGTGCAT & CATTGCGGAGTTCACAGCC & CCGATCACGGTGCTCACA \\
UBI & CACCACAGCACACGC & TTGTCCTGCGCCTCCGT & GGCATAGGTATAATGAAGTCCAATGC \\
\hline
\end{tabular}

${ }^{a}$ These probes were designed to span the introns of their genomic sequences.

The reaction mixture $(20 \mu \mathrm{L})$ contained $1.8 \mu \mathrm{L}$ of diluted cDNA, 4 pmol of each primer, 4 pmol TaqMan-MGB probe and appropriate amounts of other components as recommended by the manufacturer (Toyobo, Japan). The real-time PCR was programmed for $5 \mathrm{~min}$ at $95^{\circ} \mathrm{C}, 50$ cycles of $20 \mathrm{~s}$ at $94{ }^{\circ} \mathrm{C}$ and $30 \mathrm{~s}$ at $60{ }^{\circ} \mathrm{C}$. Each sample was quantified in triplicate. For control reactions, either no sample was added or RNA alone was added without reverse transcription to test if the RNA sample contained genomic DNA contamination. The housekeeping gene ubiquitin was used as internal standard to normalize target genes [27]. To generate standard curves for the PIPS and $U B I$, serial dilutions of the conventional RT-PCR products were made in steps of $1: 10$.

\section{$A B A$ determination}

For extraction of ABA, leaves or roots were homogenized with a mortar and pestle in an extraction solution of $80 \%$ methanol. To remove plant pigment and other non-polar compounds, which could interfere in the immunoassay, extracts were first passed through a polyvinylpyrrolidone column and C 18 cartridges. The elutants were concentrated to dryness by vacuum-evaporation and re-suspended in tris-buffered saline before enzyme-linked immunosorbent assay (ELISA). ABA content was quantified by ELISA [28]. The ABA immunoassay detection kit was purchased from the Nanjing Agriculture University.

\section{Results}

Water content and osmotic potential of leaves in upland rice and lowland rice during water deficit

Application of 20\% PEG to the root systems of both upland rice and lowland rice resulted in water deficit. The response of seedling of both upland rice and lowland rice towards water deficit was compared by analyzing the water content and osmotic potential of leaves.

Treatment with 20\% PEG rapidly and significantly decreased the water content and caused about 3\% water loss in leaves of upland rice (Figure 1A). In contrast, the water content of leaves of lowland rice reduces slightly and slowly, and only caused 1\% water loss (Figure 1A). During water deficit, the osmotic potential of leaves reduced

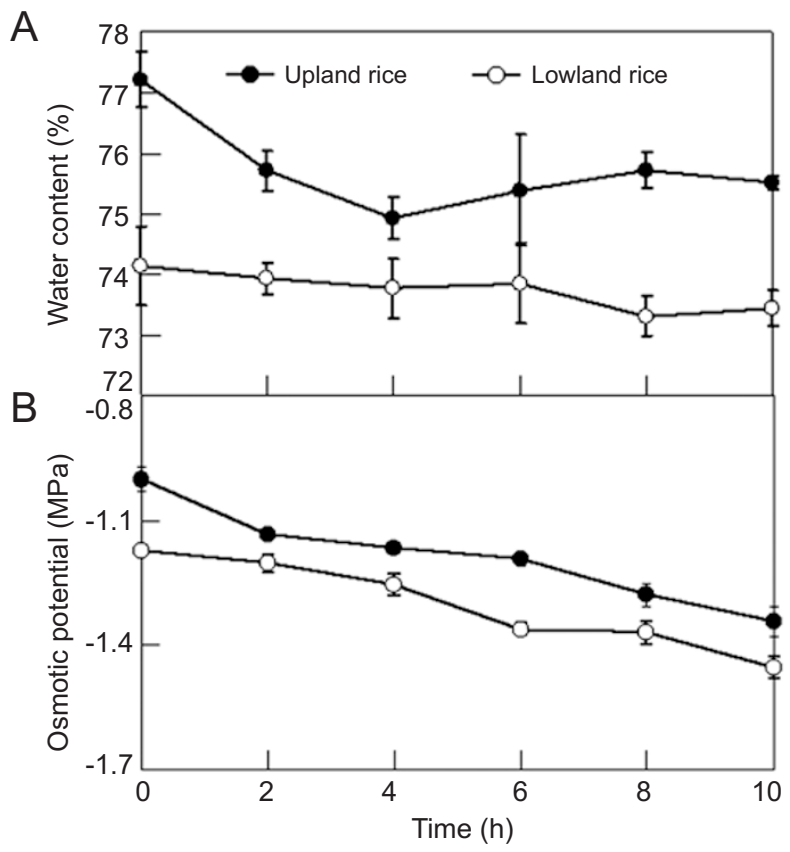

Figure 1 Effect of water deficit induced by $20 \%$ PEG 6000 on the water content (A) and osmotic potential (B) of leaves in upland rice and lowland rice. Each point is the mean of measurements $(n=3)$, and the bars represent the standard error of the mean. 
from $-1 \mathrm{MPa}$ to $-1.34 \mathrm{MPa}$ and from $-1.17 \mathrm{MPa}$ to -1.45 $\mathrm{MPa}$ in upland rice and lowland rice, respectively (Figure 1B). Within $2 \mathrm{~h}$ of PEG treatment, the osmotic potential of the leaves was strongly reduced in upland rice, but not significantly decreased in lowland rice. After the $10 \mathrm{~h}$ of PEG treatment, the osmotic potential of leaves reduced $34 \%$ and $24 \%$ in upland rice and lowland rice, respectively. These results suggested that lowland rice had better water status than upland rice when treated with 20\% PEG.

PIP protein abundance in upland and lowland rice during water deficit

The variations of PIP protein accumulation in upland rice and lowland rice under water deficit were investigated using
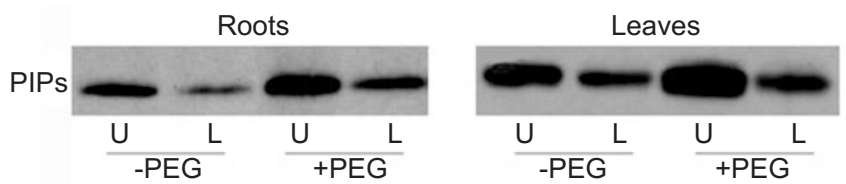

Figure 2 Immunoblot analysis of plasma membrane protein in upland rice $(\mathrm{U})$ and lowland rice $(\mathrm{L})$ under the water deficit (+ PEG) for 10 $\mathrm{h}$ or non-stressed condition (-PEG). Plasma membrane proteins (10 $\mu \mathrm{g})$ were loaded in each lane.
Western blots. The antibodies used were raised against the common N-terminal peptide of PIPs. Indeed, only one band was detected at $30 \mathrm{kDa}$ in upland rice and lowland rice. The antibodies did not cross-react with other proteins. As shown in Figure 2, upland rice had higher PIP protein abundance than lowland rice both in non-stressed conditions and after $10 \mathrm{~h}$ of $20 \%$ PEG treatment. The PEG treatment markedly enhanced the PIP protein abundance in the roots of both rice types and in leaves of upland rice. The PIP protein abundance remained approximately constant in leaves of lowland rice after $10 \mathrm{~h}$ of the water deficit treatment.

Abundance of the PIP transcripts in upland rice and lowland rice under non-stressed condition

The expression of all 11 PIP, under non-stressed conditions, was determined with real-time RT-PCR in upland rice and lowland rice. Relative transcript levels of the PIP genes were normalized by comparing them to the expression of a housekeeper gene, ubiquitin. As shown in Figure 3, PIP genes were generally transcribed at similar levels in upland rice and lowland rice, but with higher expression levels of $O S P I P 2 ; 5$ and $O S P I P 2 ; 6$ in upland rice roots. For most of the PIP genes, roots and leaves had characteristic and distinct expression levels. Many, such as $O s P I P 1 ; 2$, OsPIP1;3, OsPIP 2; 1, OsPIP2;3, OsPIP2;4, OsPIP 2;5 and $O s P I P 2 ; 6$, were expressed abundantly in roots, yet $O S$ -

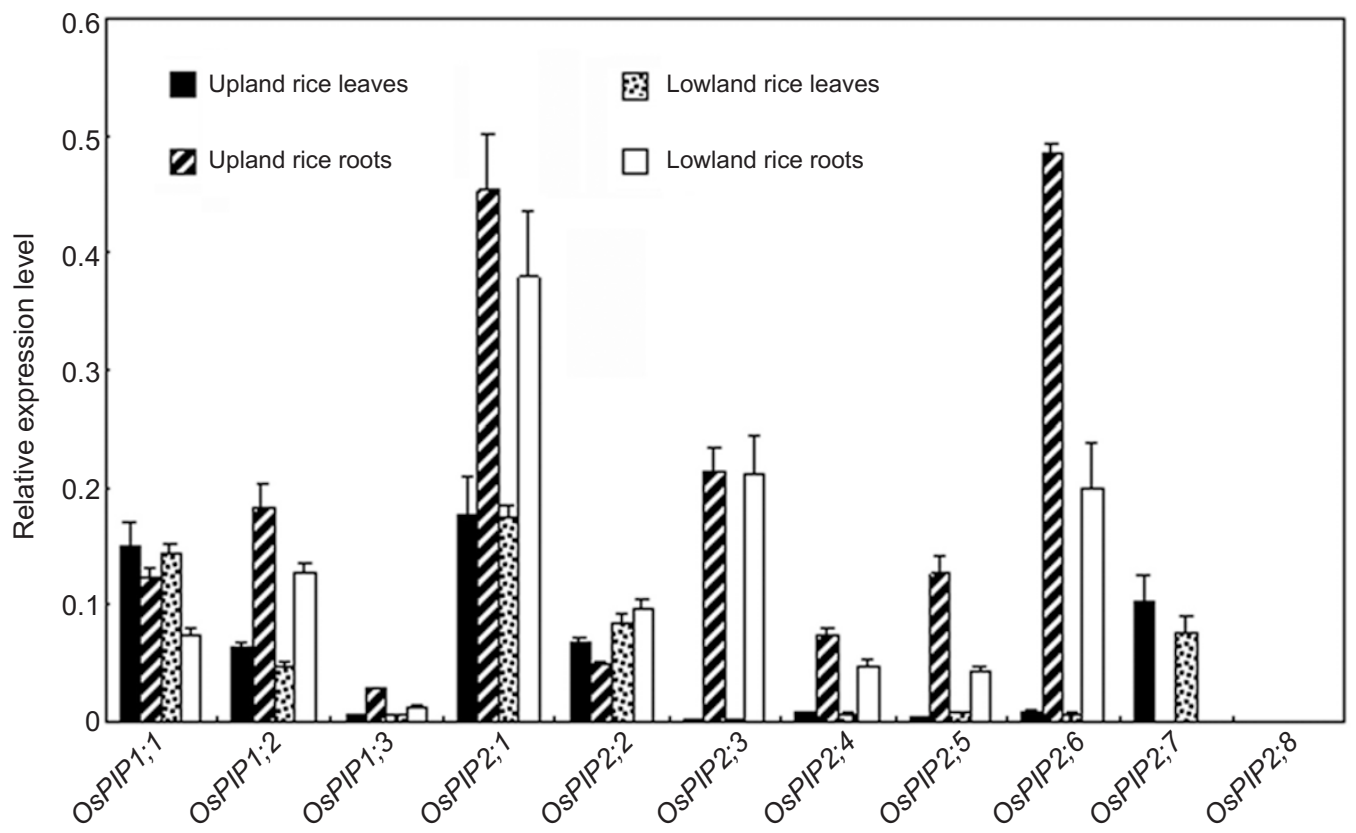

Figure 3 The transcript levels of the 11 PIPs in roots and leaves of upland rice and lowland rice. Total RNA was extracted from the roots and leaves under non-stressed condition. The transcript levels of each PIP were estimated by real-time RT-PCR and normalized to that of ubiquitin (see Materials and Methods). The values are means \pm S.D. of three replicates. 
$P I P 2 ; 7$ was more highly expressed in leaves. Furthermore, the expression of OsPIP2;7 in roots and OsPIP 2;8 were extremely low, which are $10^{-4}$ and $10^{-3}$ to that of $O S P I P 2 ; 7$ in leaves, respectively. However, very weak expression of OSPIP $2 ; 8$ could be detected by conventional RT-PCR [26]. Expression of $O s P I P 2 ; 7$ in roots and OsPIP 2;8 upon PEG and ABA treatment was not analyzed in the next experiments.

Expression profile of the PIP genes in upland rice and lowland rice during water deficit

Figure 4 shows the expression patterns of PIP genes in upland rice and lowland rice when subjected to water deficit induced by $20 \%$ PEG treatment. The expression patterns of $P I P$ genes changed in different ways in response to water deficit. The transcript level of $O s P I P 1 ; 2$ was up-regulated more than 10-fold, and that of OsPIP1;3, OsPIP2;1 and OsPIP 2;5 increased over six-fold in roots of upland rice. In contrast, no obvious enhancement in expression of these genes was observed in lowland rice under water deficit.

A

Roots
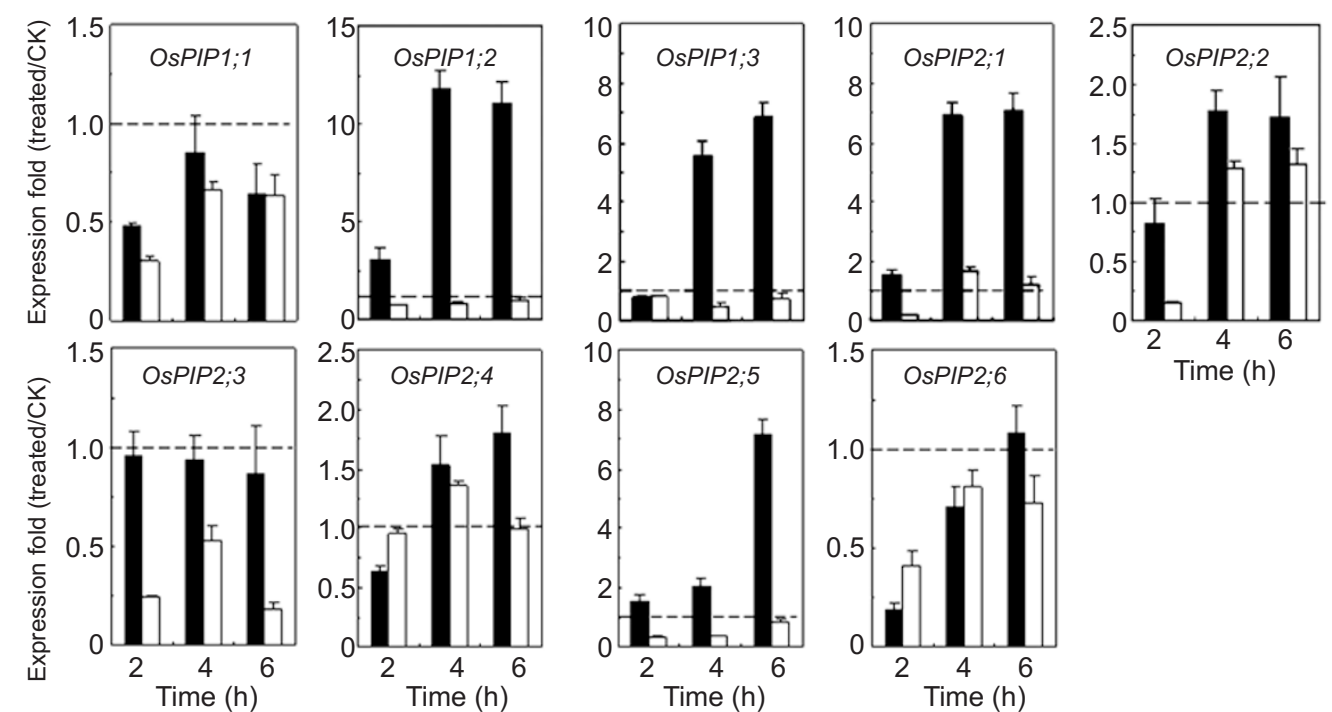

B

Leaves
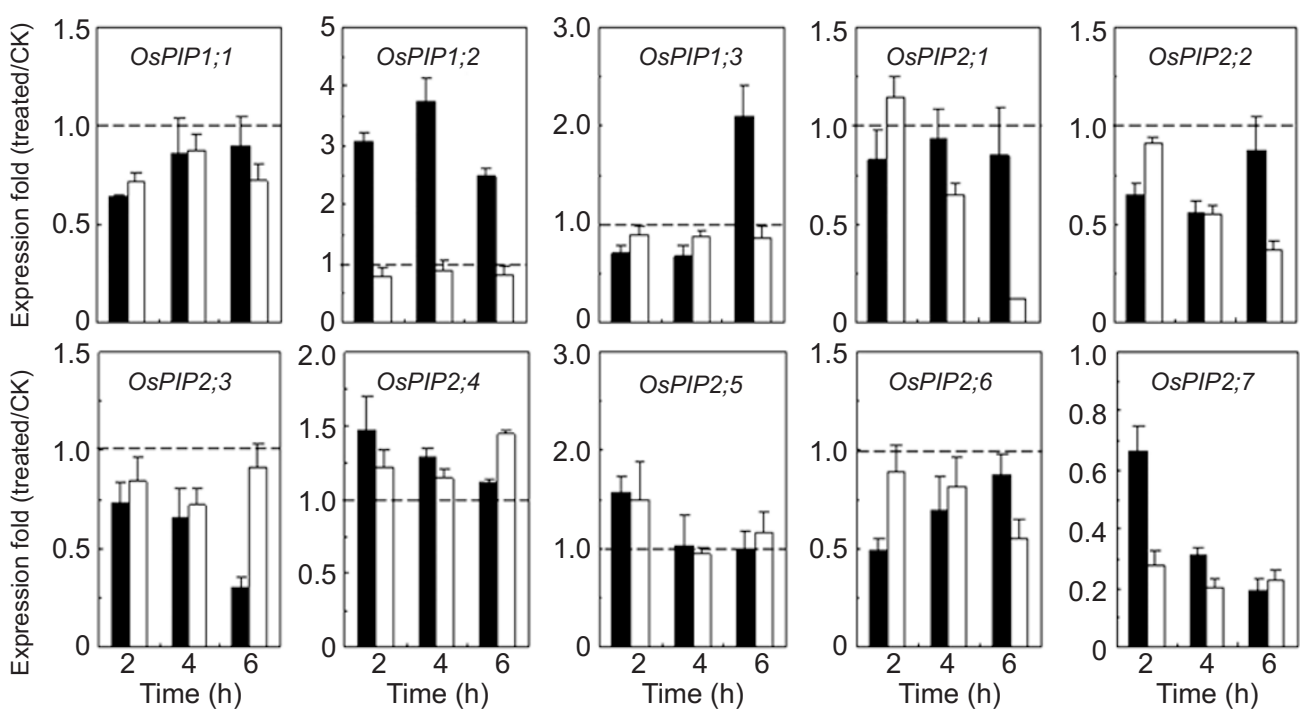

Figure 4 Relative expression of the PIP genes in upland rice (black column) and lowland rice (white column) under water deficit induced by $20 \%$ PEG 6000. The transcript levels of each PIP in the roots (A) and leaves (B) of treated plants were plotted as the relative expression (fold) of the non-stressed control plants at 2, 4 and 6 h. Values are means \pm S.D. of three replicates. 
Other genes, such as $O s P I P 1 ; 1$, and $O s P I P 2 ; 6$ in roots and OsPIP 1;1, OsPIP2;1, OsPIP2;2, OsPIP2;3, OsPIP2;6 and OsPIP 2; 7 in leaves, were down-regulated in both upland rice and lowland rice in response to the PEG treatment.

\section{ABA accumulation in upland rice and lowland rice during water deficit}

ABA acts in response to various abiotic stresses, and serves as a crucial component in the drought signaling pathway. As different responses to water stress were observed, e.g. water content and osmotic potential of leaves, PIP expression and protein levels in upland rice and lowland rice, it was of interest to determine endogenous ABA content in parallel to these changes. As shown in Figure 5, upland rice had a higher ABA level than lowland rice, especially in roots, during the $20 \%$ PEG treatment. The data clearly show that ABA increased to a maximum after $2 \mathrm{~h}$ and $4 \mathrm{~h}$ of water deficit in roots and leaves of upland rice with increases of 16.7- and 11.5-fold, respectively. After $10 \mathrm{~h}$ of water deficit, the root ABA content decreased to approximately the initial value, whereas it remained high (6.7-fold) in leaves of upland rice. In contrast, lowland rice $\mathrm{ABA}$ content did not change throughout the duration of the water deficit. Thus, ABA levels changed in parallel

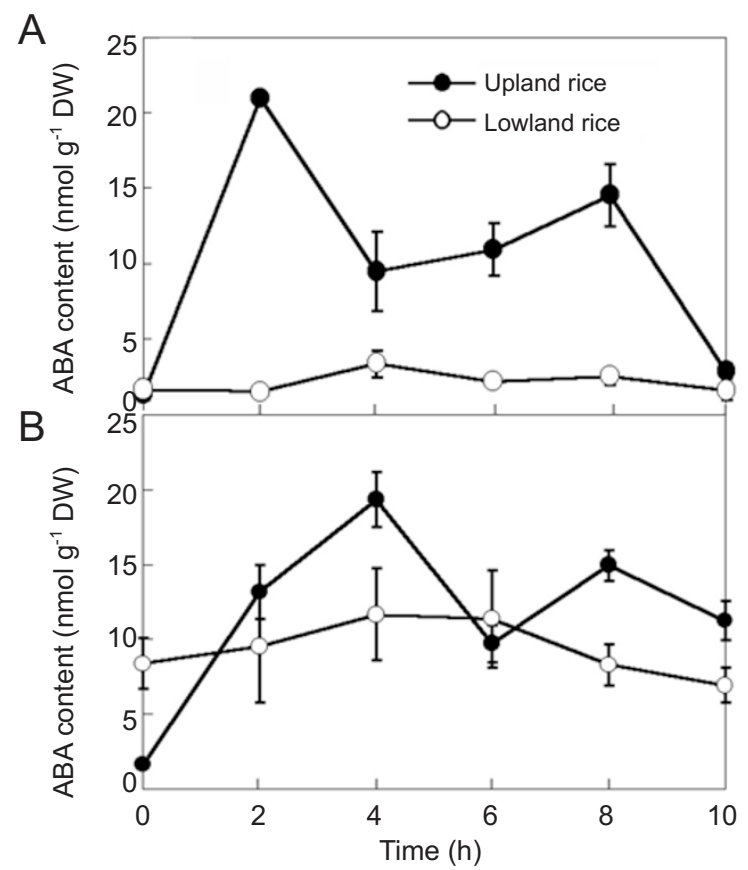

Figure 5 Change in ABA content in roots (A) and leaves (B) of upland rice and lowland rice under water deficit induced by $20 \%$ PEG 6000. Each point is the mean of measurements $(n=3)$, and the bars represent the standard error of the mean. to the changes in expression of some of the PIP genes, such as OsPIP1;2, OsPIP1;3, OsPIP 2;1 and OsPIP2;5 in roots and $O S P I P 1 ; 2, O S P I P 1 ; 3$ in leaves of upland rice in response to water deficit.

Effect of exogenous ABA on the expression of PIP genes in upland rice and lowland rice

The fact that water deficit enhanced the ABA level and the transcription of some PIP genes in upland rice (Figures 4 and 5) supports the interesting possibility that PIP expression is regulated by $\mathrm{ABA}$. In both upland rice and lowland rice, no visible difference in growth phenotype was observed between untreated and $60 \mu \mathrm{mol} \mathrm{L}{ }^{-1}$ ABA-pretreated seedlings. The transcripts of $O s P I P 1 ; 2$, OsPIP 2; 5 and OsPIP 2; 6 in roots and OsPIP1;2, OsPIP 2;4 and $O s P I P 2 ; 6$ in leaves of upland rice were up-regulated, whereas only $O S P I P 1 ; 2$ and $O S P I P 2 ; 6$ were enhanced in the roots of lowland rice (Figure 6). The up-regulation of $O S P I P 1 ; 2$, and $O S P I P 2 ; 5$ in roots as well as $O S P I P 1 ; 2$, in leaves of upland rice was reminiscent of the response to PEG treatment (Figures 4 and 6). An additional observation was that $O s P I P 2 ; 3$ and $O S P I P 2 ; 4$ in roots and $O S P I P 2 ; 2$, OsPIP 2;3 and OsPIP2; 7 in leaves were down-regulated in both upland rice and lowland rice during ABA treatment.

\section{Discussions}

Different physiological response to water stress in upland rice and lowland rice

Different physiological responses to water stress were observed in upland rice and lowland rice. Young leaf rolling was observed in the upland rice, but not in the leaves of lowland rice [21]. We also found the leaf water content and osmotic potential decreased more upland rice than lowland rice (Figure 1A and 1B). Furthermore, long-term $(7-8 \mathrm{~d})$ and mild $(-0.45 \mathrm{MPa})$ water stress also more strongly decreased the water content and osmotic potential in young upland rice (7-8 d after germination) than young lowland rice (data not shown). To reduce the efflux of water and regain turgor, lower osmotic potential in leaves is beneficial for upland rice. It was known that synthesis of compatible solute is involved in the osmoregulation [29]. Our experiment showed that PEG strongly induced the accumulation of proline in leaves of upland rice but to a lesser degree in lowland rice (data not shown). These results suggested that lowland rice had better water status under PEG treatment.

PIP expression in upland rice and lowland rice under normal condition

At the protein level, there was higher PIP accumulation in leaves than roots (Figure 2); however, this was not re- 
A Roots
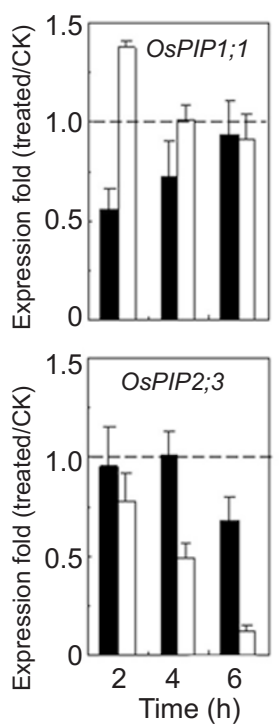

B
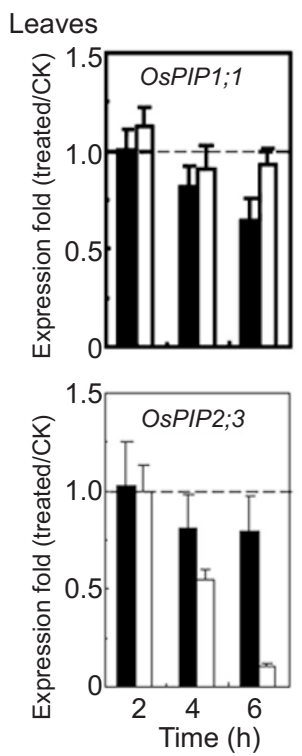
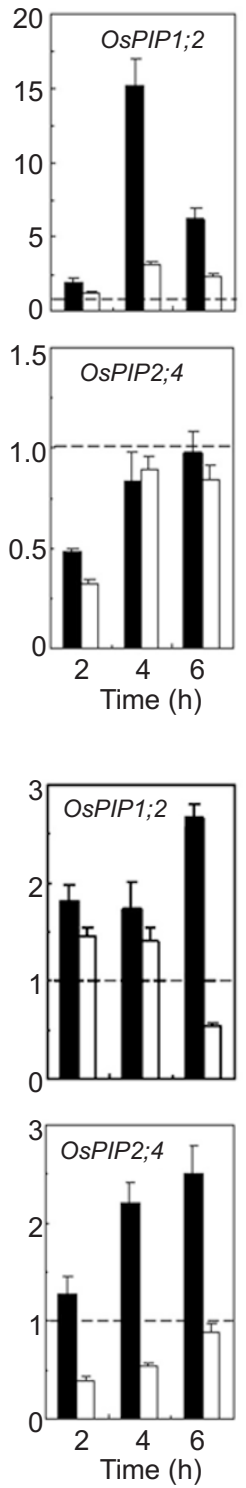
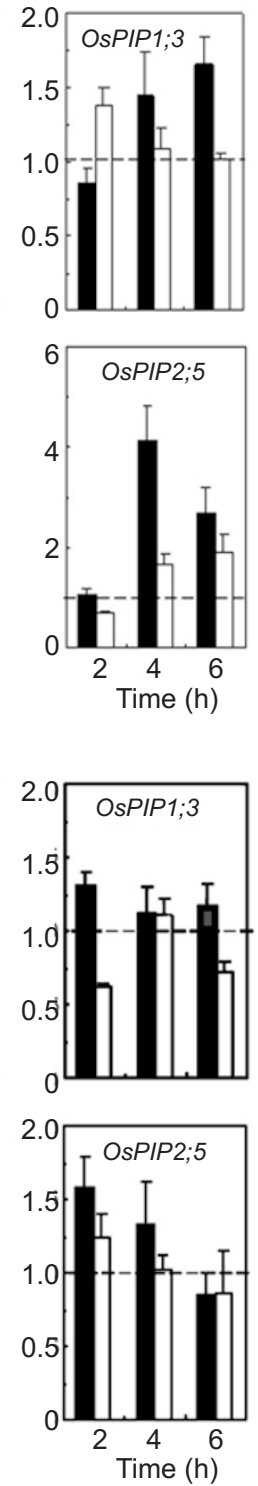
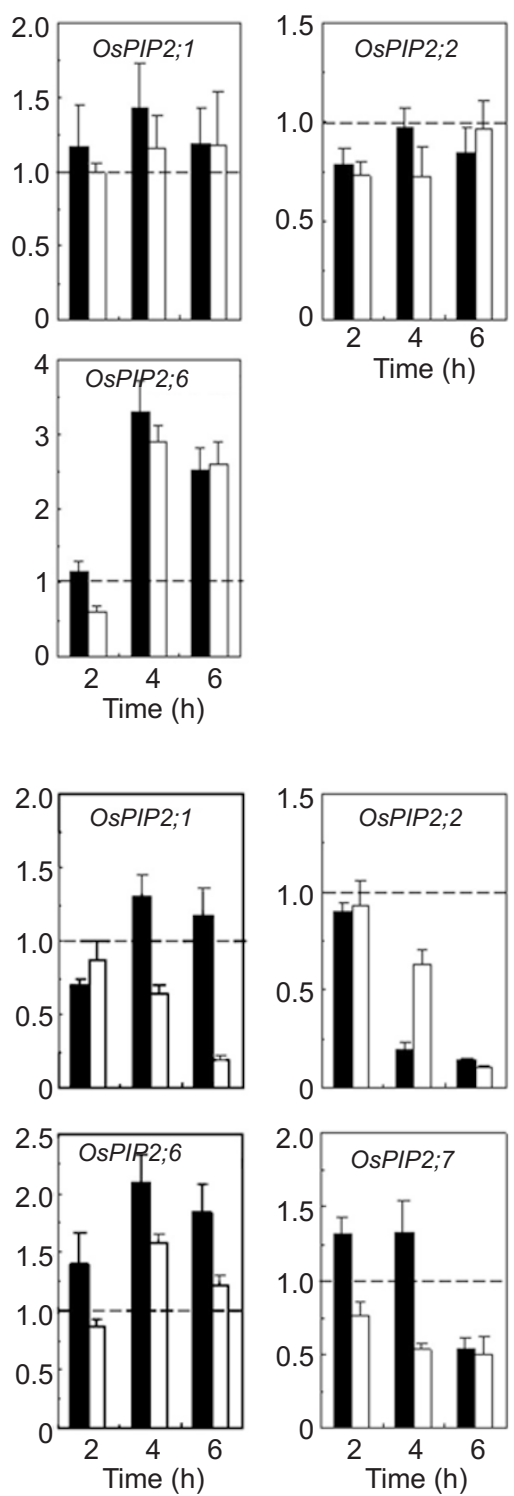

Figure 6 Effect of exogenously applied ABA on the expression of the PIP genes in upland rice (black column) and lowland rice (white column). The transcript levels of each PIP in roots (A) and leaves (B) of treated plants were plotted as the relative expression (fold) of the non-stressed control plants at 2, 4 and $6 \mathrm{~h}$. Values are means \pm S.D. of three replicates.

flected in the mRNA level measured using RT-PCR (Figure 3). Seven $P I P$ genes, i.e. OsPIP1;2, OsPIP 1;3, OsPIP 2;1, OsPIP 2;3, OsPIP2;4, OsPIP2;5 and OsPIP2;6, were transcribed at higher rates in roots than leaves and only one PIP gene, OSPIP 2; 7, followed the pattern of mRNA accumulation of higher levels in leaves than roots. Inconsistency between aquaporin expression at the mRNA level and protein accumulation was also noted in a previous study $[8,30]$. These authors postulated that post-transcriptional mechanisms resulted in this, e.g. the translation rate and degradation of PIP proteins could differentially influence PIP protein levels independently from transcription levels. Although the anti-PIP antibodies were raised against the common sequence of PIP1 and PIP2 subfamily, it is possible that some plasma membrane aquaporins were not detected, resulting in inconsistency of aquaporin expression between mRNA and protein level, due to low sequence identity in the antibody-recognizing site. Better antibodies 
that could discriminate the PIP1s and PIP2s in rice would be helpful in the future studies.

Drought-inducible PIP genes were ABA-dependent or ABA-independent

Tremendous efforts have concentrated on the expression of PIP gene in responses to drought/water deficit, and some of those were up-regulated under the stressed condition [31]. In this research, based on the integrated expression profile of $P I P$ gene family in upland rice and lowland rice during water deficit, some $P I P$ genes, i.e. OsPIP1;2, OsPIP 1;3, OsPIP2;1 and OsPIP2; 5, were also induced in upland rice (Figure 4). ABA is known to serve as a crucial component in the drought signaling pathway in plants, and induces expression of many PIPS in a number of species $[1,11,19]$. In these experiments, we also observed the induction of $O_{S P I P 1 ; 2}$ and $O S P I P 2 ; 5$ by ABA (Figure 6). Thus, ABA signaling was presumed to mediate the regulation of expression of these PIPs in upland rice during water deficit. The hypothesis was supported by the fact that an increase in ABA accumulated was parallel to some PIPs up-regulation under water deficit (Figure 5).

To date, two ABA-dependent signaling pathways that function in the activation of drought-inducible genes under water deficit have been identified $[18,32]$. One is the, bZIP/ABRE pathway and the other is mediated by the MYC/MYB transcription factors. Coincidently, when we analyzed the promoter sequence (from $-1000 \mathrm{bp}$ to initiation codon) of rice PIP genes using the software Plant Care (http://intra.psb.ugent.be:8080/PlantCARE/), the ABRE (PyACGTGGC) motif was found in OsPIP1;2 and the potential MYB recognition sequence (AACCAAA) was found in OsPIP2;5. Therefore, it was proposed that ABA regulation of $O S P I P 1 ; 2$ was via the bZIP/ABRE pathway, and that of OSPIP2;5 might be mediated by the MYC/MYB transcription factors.

However, the OsPIP1;3 and OsPIP2;1 genes in roots and $O S P I P 1 ; 3$ in leaves of upland rice were up-regulated when treated with PEG, but did not respond to ABA treatment, indicating drought-inducible expression of OsPIP1;3 and $O S P I P 2 ; 1$ were ABA-independent. These data are consistent with the response of some PIP genes in other species, such as $C p-P I P a 6, C p-P I P a 7$ and $C p-P I P c$ in $C r a-$ terostigma plantagineum [19], PIP 1;3 and PIP2;5 in Arabidopsis [11] and TRAMP in tomato [6]. It has been shown that some of the drought-responsive genes are induced in an ABA-independent pathway, and the dehydration-responsive-element/C-repeat (DRE/CRT) cis-element was found in the promoter region of these genes [32]. Analysis of the promoter sequences of OSPIP1;3 and OSPIP 2;1 (http://intra.psb.ugent.be:8080/PlantCARE/) resulted in the identification of two putative DRE/CRT boxes (TACC-
GACAT) in the OsPIP 1;3 promoter at -239 bp and -721 bp, respectively. No DRE/CRT was found in the OsPIP2;1 promoter region from $-2000 \mathrm{bp}$ to the initiation codon. Thus, the up-regulation of $O S P I P 1 ; 3$ under water deficit was probably via the DRE/CRT-dependent pathway.

\section{Roles of PIPs in rice drought avoidance}

Although important progress has been made in understanding the role of aquaporins in plant adaptations to dehydration condition, opinions are still in conflict. One point of view is that an increase in aquaporin level results in higher membrane water permeability and facilitates water transport $[6,7,33,34]$. Overexpression of BnPIP1 in transgenic tobacco plants also increased tolerance to water stress at the whole plant level, and partial silencing of $B n P I P 1$ resulted in reduced water uptake and decreased tolerance to water stress [34]. A second point of view is that plants decrease their membrane water permeability to avoid excessive loss of water by down-regulation of some aquaporins under water deficit $[9,35,36]$. For instance, Aharon et al. [36] constitutively over-expressed the Arabidopsis plasma membrane aquaporin PIP1b in tobacco, and these transgenic plants wilted more quickly during water stress.

In a previous study, upland rice and lowland rice were characterized as drought avoidance and drought tolerance, respectively [21]. So the comparison of upland rice and lowland rice appears to be a paradigm for studying the role of aquaporins in drought resistance. The fact that the upland rice had deeper, wider roots (data not shown) as well as higher levels of PIP protein than lowland rice (Figure 2) might be beneficial to absorb more, deeper underground water in order to supply adequate water for aerial parts when drought occurs. At the mRNA level, we observed that upland rice up-regulated $O_{S} P I P 1 ; 2, O_{s} P I P 1 ; 3, O_{S P I P} ; 1$ and $O_{s} P I P 2 ; 5$ in roots and $O s P I P 1 ; 2$ and $O s P I P 1 ; 3$ in leaves (Figure 4 ), and that this matched the changes in the amount of total PIP protein. On the other hand, the reduced transcript levels of $O S P I P 1 ; 3$ and $O S P I P 2 ; 5$ in roots of lowland rice (Figure 3 ) imply that a totally different drought tolerance mechanism may operate in this rice type. The reduction of aquaporins in lowland rice may inhibit membrane water permeability, thereby avoiding excessive loss of cellular water under water deficit. It appears that these seemingly opposite aquaporin gene regulation responses in upland rice and lowland rice, under water deficit, reflect different adaptive mechanisms for dealing with water deficit.

Subsequently, a question arose as to why the water deficit with $20 \%$ PEG treatment reduced the expression of these PIP genes (for instance, OsPIP1;3 and OsPIP 2;5 in roots) in lowland rice, whereas enhanced it in upland 
rice. This probably could be explained by the different drought-sensitive between upland rice and lowland, although other mechanisms could not be ruled out. Upland rice was drought-sensitive and lowland rice was droughttolerant crop under PEG treatment [21]. Namely, 20\% PEG treatment was a severe water stress for upland rice, whereas only a mild stress for lowland rice. It was proposed that mild water stress caused the down-regulation of PIP expression, whereas more severe drought caused PIP upregulation [7].

Some PIP genes in our experiments (OsPIP $1 ; 1, O_{s}-$ $P I P 2 ; 6$ in roots and OsPIP2;3, OsPIP 2;7 in leaves) were down-regulated in upland rice (Figure 4 ). We also observed that the transcript level of OsPIP2;4 in roots as well as some PIP genes in leaves of lowland rice remained unchanged under water deficit (Figure 3 ). These observations are not explained by the above-mentioned points, but in Arabidopsis, up- or down-regulation of PIP genes under water deficit was also described [11, 12]. This diversity of PIP expression patterns in plants implies that the role of aquaporins in drought resistance is influenced by many factors. Owing to the complexity of the response of PIPs, a comprehensive understanding of these proteins in response to drought resistance remains a challenge to be explained by future research.

\section{Acknowledgments}

We thank Dr M Maeshima for the anti-PIP antibodies. This work is supported by the Key Basic Research Special Fund of CAS (KSCXZ-SW-116).

\section{References}

1 Tyerman SD, Bohnert HJ, Maurel C, Steudle E, Smith JAC. Plant aquaporins: their molecular biology, biophysics and significance for plant water relations. J Exp Bot 1999; 50:1055-1071.

2 Johansson I, Karlsson M, Johanson U, Larsson C, Kjellbom P. The role of aquaporins in cellular and whole plant water balance. Biochim Biophys Acta 2000; 1465:324-342.

3 Javot H, Maurel C. The role of aquaporin in root water uptake. Ann Bot 2002; 90:301-313.

4 Luu DT, Maure C. Aquaporins in a challenging environment: molecular gears for adjusting plant water status. Plant Cell Environ 2005; 28:85-96

5 Malz S, Sauter M. Expression of two PIP genes in rapidly growing internodes of rice is not primarily controlled by meristem activity or cell expansion. Plant Mol Biol 1999; 40:985-995.

6 Fray RG, Wallace A, Grierson D, Lycett GW. Nucleotide sequence and expression of a ripening and water stress-related cDNA from tomato with homology to the MIP class of membrane channel proteins. Plant Mol Biol 1994; 24:539-543.

7 Yamada S, Komori T, Myers PN, et al. Expression of plasma membrane water channel genes under water stress in Nicotiana excelsior. Plant Cell Physiol 1997; 38:1226-1231.
8 Suga S, Komatsu S, Maeshima M. Aquaporin isoforms responsive to salt and water stresses and phytohormones in radish seedlings. Plant Cell Physiol 2002; 43:1229-1237.

9 Smart LB, Moskal WA, Cameron KD, Bennett AB. MIP genes are down-regulated under drought stress in Nicotiana glauca. Plant Cell Physiol 2001; 42:686-693.

10 Li LG, Li S, Tao Y, Kitagawa Y. Molecular cloning of a novel water channel from rice: its products expression in Xenopus oocytes and involvement in chilling tolerance. Plant Sci 2000; 154:43-51.

11 Jang JY, Kim DG, Kim YO, Kim JS, Kang H. An expression analysis of a gene family encoding plasma membrane aquaporins in response to abiotic stresses in Arabidopsis thaliana. Plant Mol Biol 2004; 54:713-725

12 Alexandersson E, Fraysse L, Sjövall-Larsen S, et al. Whole gene family expression and drought stress regulation of aquaporins. Plant Mol Biol 2005; 59:469-484.

13 Morillon R, Chrispeels J. The role of ABA and the transpiration stream in the regulation of the osmotic water permeability of leaf cells. Proc Natl Acad Sci USA 2001; 98:14138-14143.

14 Wan X, Zwiazek JJ. Root water flow and leaf stomatal conductance in aspen (Populus tremuloides) seedlings treated with abscisic acid. Planta 2001; 213:741-747.

15 Quintero J, Fournier J, Benlloch M. Water transport in sunflower root systems: effects of ABA, $\mathrm{Ca}^{2+}$ status and $\mathrm{Hgcl}_{2}$. J Exp Bot 1999; 50:1607-1612.

16 Hose E, Steudle E, Hartung W. Abscisic acid and hydraulic conductivity of maize roots: a study using cell-and root-pressure probes. Planta 2000; 211:874-882.

17 Bray EA. Plant responses to water deficit. Trends Plant Sci 1997; 2:48-54.

18 Shinozaki K, Yamaguchi-Shinozaki K. Molecular responses to dehydration and low temperature: differences and cross-talk between two stress signaling pathways. Curr Opin Plant Biol 2000; 3:217-223

19 Mariaux JB, Bockel C, Salamini F, Bartel D. Desiccation- and abscisic acid-responsive genes encoding major intrinsic proteins (MIPs) from the resurrection plant Craterostigma plantagineum. Plant Mol Biol 1998; 38:1089-1099.

20 Zhu CF, Schraut D, Hartung W, Schaffner AR. Differential responses of maize MIP genes to salt stress and ABA. J Exp Bot 2005; 56:2971-2981

21 Lian HL, Yu X, Ye Q, et al. The role of aquaporin RWC3 in drought avoidance in rice. Plant Cell Physiol 2004; 45:481489.

22 Ni JS. Solution culture of rice. In: Xue YL, Xia ZA, eds. Experiment Handbook of Plant Physiology. Shanghai: Science Press 1985:63-65.

23 Ohshima Y, Iwasaki I, Suga S, et al. Low aquaporin content and low osmotic water permeability of the plasma and vacuolar membranes of a CAM plant Graptopetalum paraguayense: comparison with radish. Plant Cell Physiol 2001; 42:11191129.

24 Bradford MM. A rapid and sensitive for the quantitation of microgram quantitites of protein utilizing the principle of proteindye binding. Anal Biochem 1976; 72:248-254.

25 Sambrook F, Fritsch EF, Maniatis T. Molecular Cloning: A Laboratory Manual. 2nd Edition. Cold Spring Harbor, NY: Cold Spring Harbor Press, 1989. 
26 Sakurai J, Ishikawa F, Yamaguchi T, Uemura M, Maeshima M. Identification of 33 Rice aquaporin genes and analysis of their expression and function. Plant Cell Physiol 2005; 46:15681577.

27 Nishi R, Hashimoto H, Kidou S, Uchimiya H, Kato A. Isolation and characterization of a rice cDNA which encodes a ubiquitin protein and a 52 amino acid extension protein. Plant Mol Biol 1993; 22:159-161.

28 Walker-Simmons M. ABA levels and sensitivity in developing wheat embryos of sprouting resistant and susceptible cultivars. Plant Physiol 1987; 84:61-66.

29 Delauney AJ, Verma DPS. Proline biosynthesis and osmoregulation in plants. Plant J 1993; 4:215-223.

30 Kammerloher W, Fischer U, Piechottka GP, Schaffner AR. Water channels in the plant plasma membrane cloned by immunoselection from a mammalian expression system. Plant J 1994; 6:187-199.

31 Tyerman SD, Niemietz CM, Bramley H. Plant aquaporins: multifunctional water and solute channels with expanding roles. Plant Cell Environ 2002; 25:173-194.

32 Shinozaki K, Yamaguchi-Shinozaki K. Gene expression and signal transduction in water-stress response. Plant Physiol 1997; 115:327-334.
33 Johansson I, Karlsson M, Shukla VK, et al. Water transport activity of the plasma membrane aquaporin PM28A is regulated by phosphorylation. Plant Cell 1998; 10:451-459.

$34 \mathrm{Yu}$ QJ, Hu YL, Li JF, Wu Q, Lin ZP. Sense and antisense expression of plasma membrane aquaporin BnPIP1 from Brassica napus in tobacco and its effects on plant drought resistance. Plant Sci 2005; 169:647-656.

35 Yamada S, Katsuhara M, Kelly WB, Michalowski CB, Bohnert HJ. A family of transcripts encoding water channel proteins: tissue-specific expression in the common ice plant. Plant Cell 1995; 7:1129-1142.

36 Aharon R, Shahak Y, Wininger S, et al. Overexpression of a plasma membrane aquaporin in transgenic tobacco improves plant vigor under favorable growth conditions but not under drought or salt stress. Plant Cell 2003; 15:439-447.

37 Boursiac Y, Chen S, Luu DT, et al. Early effects of salinity on water transport in Arabidopsis roots. Molecular and cellular features of aquaporin expression. Plant Physiol 2005; 139:790805 .

Edited by Lu Liang 